\title{
Endoscopic foraminotomy using a microendoscopic discectomy system in cadaveric specimens
}

Sung Woo Roh, M.D., Daniel H. Kim, M.D., Alberto C. Cardoso, M.D., and Richard G. Fessler, M.D., Ph.D.

Department of Neurosurgery, University of Florida, Gainesville, Florida

Although the anterior approach is more commonly performed for the treatment of cervical disc disease, the posterior approach has distinct advantages in selected cases of foraminal stenosis and posterolateral disc herniation. The authors performed cervical key hole foraminotomies using a microendoscopic discectomy (MED) system in four cadaveric cervical spine specimens to evaluate this minimally invasive surgical approach for cervical disc diseases. The amount of bone decompression achieved by using the MED system was compared with that achieved by using the open foraminotomy procedure in each cadaveric specimen. Three noncontiguous cervical nerve roots were selected between C-3 and C-8 in each specimen and were decompressed using the MED system on one side and using the open foraminotomy procedure on the contralateral side. Postoperative computerized tomography (CT) myelography showed that adequate bone decompression was achieved by using either the MED or open procedure in all specimens. Postoperatively, open dissection was performed to confirm and compare the amount of decompression in both the MED and open procedures. The laminotomy size (vertical and transverse diameter), the length of decompressed nerve root, and the proportion of removed facet joint were measured on every operative level. The average vertical diameter of laminotomy area and the percentage of facet removed were significantly greater in the MED procedure than the open procedure $(\mathrm{p}$ $<0.05)$. The transverse diameter of the laminotomy area and the average decompressed root length were not significantly different between MED and open surgery. The authors conclude that endoscopic cervical foraminotomy using the MED system is a feasible procedure and may be clinically applicable in the treatment of foraminal stenosis and laterally located cervical disc herniation.

Key Words * posterior cervical foraminotomy * MED system * foraminal stenosis * cervical disc herniation

Various anterior and posterior operative approaches for the treatment of cervical disc diseases have been described.[1-3,8,9,14,15,18,19] Since the development of posterior cervical disc surgery by Scoville, several retrospective studies have been published confirming the efficacy of this procedure.[1,5,7-12,15,16,18,19-21] Although the anterior approach has become more popular than the posterior approach, posterior foraminotomy is an effective procedure in selected cases of posterolateral disc herniation and cervical foraminal stenosis. However, whereas some surgeons use small incisions and 
minimal dissection techniques in this posterior procedure, it is usually necessary to make moderate-sized incisions and significant paraspinous muscle dissection.[1,18] Postoperative wound pain, therefore, can be significant, and postoperative recovery is often relatively slow.

Among the various current minimally invasive spine surgery techniques, the microendoscopic discectomy (MED) system is a newly developed endoscope system that enables posterior lumbar discectomy through a tubular retractor under endoscopic visualization (unpublished data). The present study was performed to evaluate the technical feasibility and efficacy of the MED system for posterior cervical foraminotomy.

\section{CLINICAL MATERIAL AND METHODS}

The cervical spines of four human cadavers were used for this study. The age at the time of death ranged from 62 to 70 years. Posterior cervical foraminotomy was performed on the left side by using the MED technique and on the right side by using the open procedure. Each procedure was used to decompress 12 nerve roots. The left C-4, C-6, and C-8 levels were decompressed in two cadavers, and the left C-3, C-5 and C-7 levels were decompressed in the other two cadavers by using the MED system. The 12 contralateral nerve roots were decompressed by using the open procedure. Under fluoroscopic guidance, a guide wire was inserted through the posterior cervical musculature with the tip directed to the disc space. Three dilators were sequentially inserted through posterior neck musculature at the junction of lamina and lateral mass. A 16-mm tubular retractor was inserted over the largest dilator, fixed to the flexible arm assembly on the table, and the endoscope was then fixed inside the tubular retractor. The tubular retractor tip was placed to expose the lateral portion of superior and inferior laminae and the medial portion of the corresponding facet joint. Once exposure had been achieved, a small curved curette was used to define the edge of the superior lamina and the lateral mass. Bone removal began on the inferolateral portion of the superior lamina and proceeded on to the superolateral portion of inferior lamina. After the medial edge of the superior and inferior pedicles was palpated using a microprobe, bone removal was extended into the medial facet joint to expose the nerve root. Bone removal was performed with small Kerrison punch or high-speed drill. The surgical procedure was concluded after exposing the proximal portion of nerve root and confirming that adequate bony decompression had been achieved.[4,6,22] In each specimen pre- and postoperative CT myelography was performed to evaluate and compare the amount of bony decompression between MED and open surgery. After surgery was completed, open dissection was performed on all specimens, and the authors measured the amount of bony decompression (vertical and transverse diameters of laminotomy defect), length of root decompressed, and the proportion of facet joint removed. All measurements were statistically compared using Student's t-test.

\section{RESULTS}

Adequate exposure of the surgical area (portion of superior, inferior lamina, and medial facet joint) was possible with the 16-mm tubular retractor endoscope in all cadaver specimens (Fig. 1). 


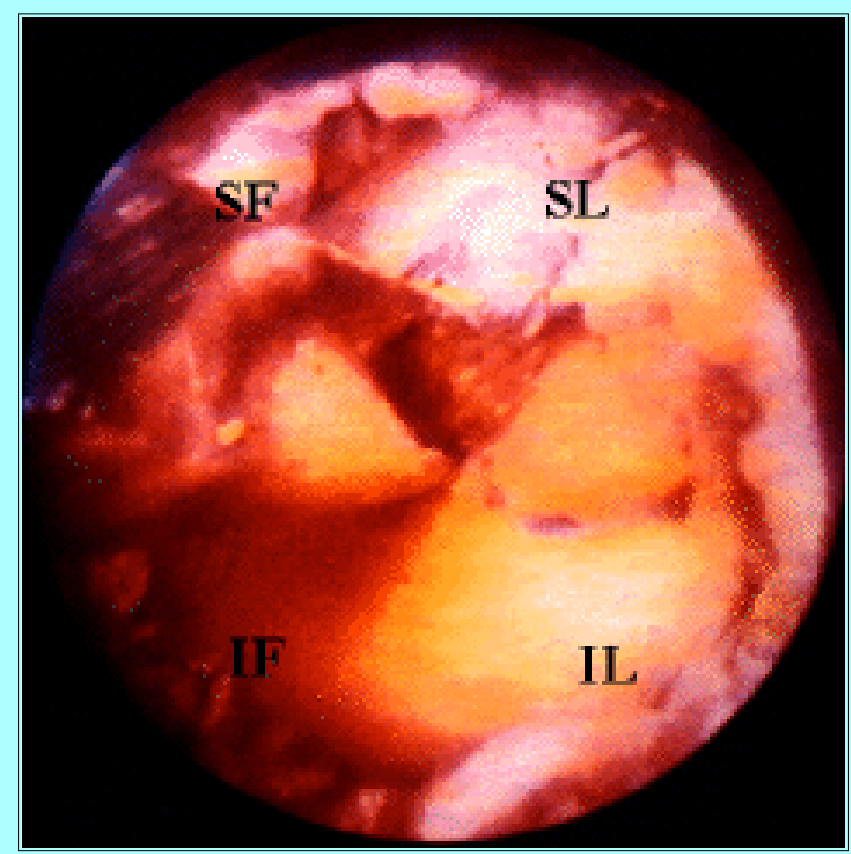

Fig. 1. Intraoperative endoscopic view through a $16-\mathrm{mm}$ tubular retractor. IF $=$ inferior facet; $\mathrm{IL}=$ inferior lamina; $\mathrm{SF}=$ superior facet; $\mathrm{SL}=$ superior lamina.

Compared with open surgery, the duration of surgery was longer in MED cases. Laminotomy size in MED surgery tended to be slightly larger than open surgery, but postoperative CT myelography showed adequate decompression on every level in both MED and open surgery (Fig. 2).

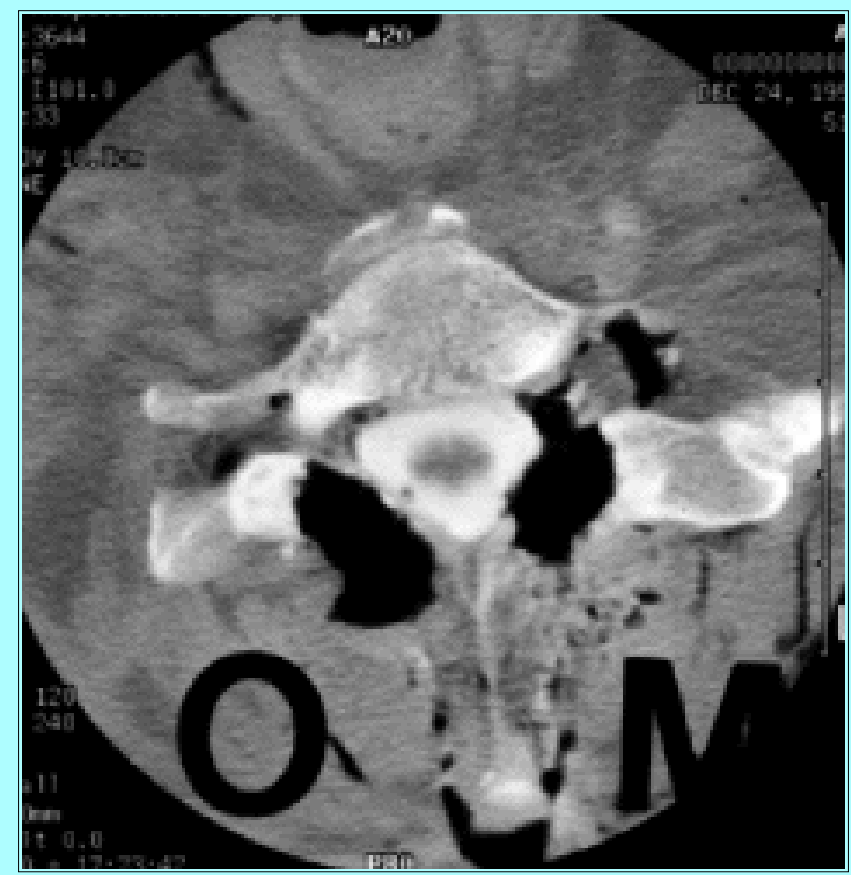

Fig. 2. Postoperative CT myelogram demonstrating adequate decompression by using both MED and open procedures. $\mathrm{O}=$ open; $\mathrm{M}=\mathrm{MED}$.

Measurements after postoperative open dissection showed that the average vertical diameter of laminotomy defect was $12 \pm 3.6 \mathrm{~mm}$ for MED and $9.6 \pm 1.9 \mathrm{~mm}$ for open laminotomy. This difference was significant $(\mathrm{t}=2.1, \mathrm{df}=22, \mathrm{p}<0.05)$. Transverse diameters (MED $13.5 \pm 2.9 \mathrm{~mm}$, open $11.5 \pm 1.9$ $\mathrm{mm}$ ) were not statistically significantly different. An average of $37.5 \pm 12 \%$ was removed by using the MED technique compared with $32.8 \pm 6 \%$ removed by using the open technique. This result was also 
significantly different $(\mathrm{t}=1.2, \mathrm{df}=22, \mathrm{p}<0.05)$. Results for length of nerve root exposed (MED $5.1 \pm$ $1.4 \mathrm{~mm}$, open $4.3 \pm 1 \mathrm{~mm}$ ) were not significantly different (Table 1). Postoperatively there was one open surgery case $(8.3 \%)$ dural sac laceration on the MED side and no dural sac or nerve root injury on the open side.

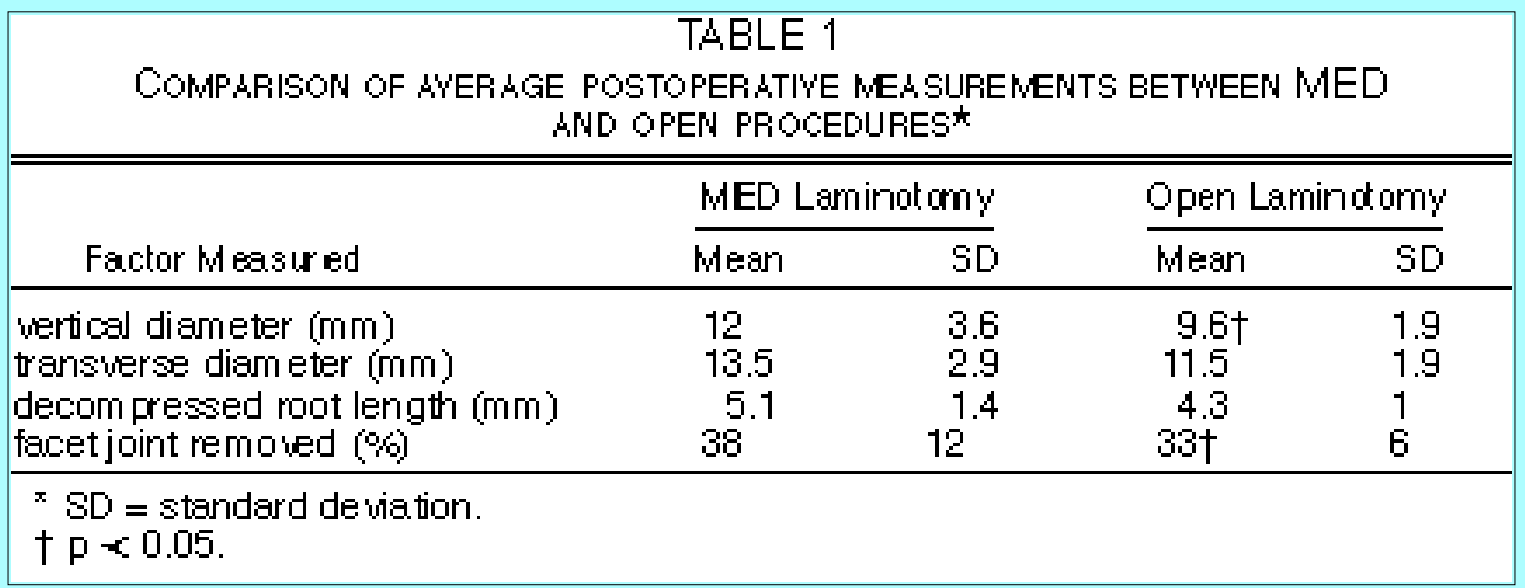

\section{DISCUSSION}

Although the anterior surgical approach to most nontraumatic cervical disc disease has been the preferred choice for most spine surgeons, previously reported studies have demonstrated similar operative results in both anterior and posterior surgical approaches.[1-3,5,7-10,12,14-16,18,19-21] In some cases, such as laterally herniated discs causing radiculopathy, there are distinct advantages to the posterior approach. The posterior approach via key hole foraminotomy provides significantly greater exposure of the exiting nerve root for decompression and removal of laterally herniated disc.[17] Compared with the anterior approach, use of the posterior approach can avoid causing surgical injuries to carotid arteries, recurrent laryngeal nerves, and other prevertebral structures. The posterior surgical approach with minimal medial facetectomy also eliminates the need for fusion.[23] The major disadvantage of current posterior surgical approaches is postoperative cervical muscular pain and spasm, resulting from subperiosteal dissection of paraspinal muscles. This may even prolong postoperative hospitalization.

In this study we evaluated the feasibility of using a minimally invasive posterior cervical endoscopic technique to provide adequate foraminal decompression and discectomy. Transmuscular dilation by using the endoscopic tubular working channel can minimize postsurgical pain and provide rapid posoperative recovery.

During cervical foraminotomy performed using the MED system, localization of correct surgical level can be achieved with a guide wire under fluoroscopic anteroposterior and lateral guidance. The skin incision is usually made $0.5 \mathrm{~cm}$ from the midline, and the tubular working channel is positioned to expose the lateral portion of the superior and inferior lamina and the medial portion of the corresponding facet joint. Bone decompression is achieved by using conventional open key hole foraminotomy techniques. Minimal skin incision, but no muscle dissection, is required.

The results of postoperative open dissection showed that average vertical and transverse diameters of laminotomy defects were not significantly different, or were greater, using the MED technique. The length of decompressed nerve roots and the average proportion of facet joint removed was also not significantly different or was greater using the MED technique. These results clearly suggest that MED may provide a viable technique for cervical hemilaminectomy and foraminotomy. Results are at least 
equivalent to the open technique. Adjacent levels above and below can be also visualized and decompressed by manipulation and changing the angle of the endoscopic working channel. Similar to the open technique, posterior key hole foraminotomy is indicated for foraminal stenosis and lateral disc herniations. Central and paracentral cervical disc herniations should be approached anteriorly.

One question we could not answer in this study was whether it is feasible to control hemostasis adequately from the epidural venous plexus by using this technique. Experience with lumbar MED has indicated that hemostasis can, at times, be challenging and because the epidural venous plexus surrounding the cervical nerve roots can cause annoying bleeding, this is an important issue.[13] Studies in live porcine animals are currently underway to answer this question.

\section{CONCLUSION}

The feasibility of adequate bone decompression and exposure of cervical nerve roots by the MED system has been well demonstrated in this study in which human cadaveric specimens were used. In addition to evaluating the ability of MED to control hemostasis, research is currently in progress to refine instrumentation and surgical technique for this procedure.

\section{References}

1. Aldrich F: Posterolateral microdiscectomy for cervical monoradiculopathy caused by posterolateral soft cervical disc sequestration. J Neurosurg 72:370-377, 1990

2. Aronson NN: The management of soft cervical disc protrusions using the Smith-Robinson approach. Clin Neurosurg 20:253-258, 1973

3. Bailey RW, Badgley CE: Stabilization of the cervical spine by anterior fusion. J Bone Joint Surg (Am) 42:565-594, 1960

4. Ball PA: Management of cervical disc disease (posterior approach), in Menezes AH, Sonntag VKH (eds): Principles of Spinal Surgery. New York: McGraw-Hill, 1996, Vol 1, pp 539-546

5. Davis RA: A long-term outcome study of 170 surgically treated patients with compressive cervical radiculopathy. Surg Neurol 46:523-533, 1996

6. Dick P: Cervical foraminotomy: indications and technique, in Watkins RG (ed): Surgical Approaches to the Spine. New York: Springer-Verlag, 1983, pp 140-147

7. Dillin W, Booth R, Cuckler J, et al: Cervical radiculopathy. A review. Spine 11:988-991, 1986

8. Fager CA: Management of cervical disc lesions and spondylosis by posterior approaches. Clin Neurosurg 24:488-507, 1977

9. Fager CA: Posterolateral approach to ruptured median and paramedian cervical disk. Surg Neurol 20:443-452, 1983

10. Henderson CM, Hennessy RG, Shuey HM Jr, et al: Posterior-lateral foraminotomy as an exclusive operative technique for cervical radiculopathy: a review of 846 consecutively operated cases.

Neurosurgery 13:504-512, 1983

11. Herkowitz HN, Kurz LT, Overholt DP: Surgical management of cervical soft disc herniation. A 
comparison between the anterior and posterior approach. Spine 15:1026-1030, 1990

12. Krupp W, Schattke H, Muke R: Clinical results of the foraminotomy as described by Frykholm for the treatment of lateral cervical disc herniation. Acta Neurochir 107:22-29, 1990

13. Kubo Y, Waga S, Kojima T, et al: Microsurgical anatomy of the lower cervical spine and cord. Neurosurgery 34:895-902, 1994

14. Manabe S, Tateishi A, Ohno T: Anterolateral uncoforaminotomy for cervical spondylotic myeloradiculopathy. Acta Orthop Scand 59:669-674, 1988

15. Mosdal C, Overgaard J: Lateral cervical facetectomy. The surgical pathology of radicular brachialgia. Acta Neurochir 70:199-205, 1984

16. Murphey F, Simmons JCH, Brunson B: Surgical treatment of laterally ruptured cervical disc. Review of 648 cases, 1939 to 1972. J Neurosurg 38:679-683, 1973

17. Raynor RB: Anterior or posterior approach to the cervical spine: an anatomical and radiographic evaluation and comparison. Neurosurgery 12:7-13, 1983

18. Scoville WB, Dohrmann GJ, Corkill G: Late results of cervical disc surgery. J Neurosurg 45:203-210, 1976

19. Tomaras CR, Blacklock B, Parker WD: Outpatient surgical treatment of cervical radiculopathy. J Neurosurg 87:41-43, 1997

20. Williams RW: Microcervical foraminotomy. A surgical alternative for intractable radicular pain. Spine 8:708-716, 1983

21. Woertgen C, Holzschuh M, Rothoerl RD, et al: Prognostic factors of posterior cervical disc surgery: a prospective, consecutive study of 54 patients. Neurosurgery 40:724-729, 1997

22. Young PH: Microsurgery of the Cervical Spine. New York: Raven Press, 1991, pp 143-148

23. Zdeblick TA, Zou D, Warden KE, et al: Cervical stability after foraminotomy. A biomechanical in vitro analysis. J Bone Joint Surg (Am) 74:22-27, 1992

Manuscript received January 16, 1998.

Accepted in final form January 27, 1998.

Address reprint requests to: Richard Fessler, M.D., Ph.D., Department of Neurological Surgery, College of Medicine, University of Florida, P.O. Box 100265, Gainesville, Florida 32610-0265. 